\title{
NAOMI GAZL: A MULTISPECIES DIAL TESTED ON THE TADI GAS LEAK SIMULATION FACILITY
}

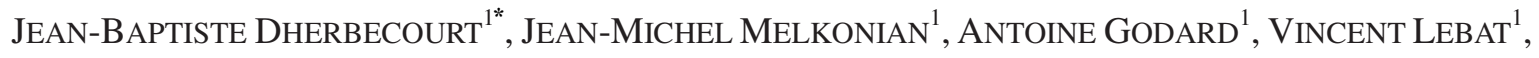 \\ NiCOLAS TANGUY ${ }^{1}$, CEDRIC BLANCHARD ${ }^{1}$, STÉPHANIE DOZ ${ }^{3}$, PIERRE-YvES FOUCHER ${ }^{3}$, THIERRY HUET ${ }^{3}$, \\ XAVIER WATREMEZ ${ }^{2}$, DOMINIQUE DUBUCQ ${ }^{2}$, MYRIAM RAYBAUT ${ }^{1 *}$ \\ ${ }^{1}$ DPHY, ONERA, Université Paris Saclay, F-91123 Palaiseau, France \\ ${ }^{2}$ TOTAL, France \\ ${ }^{3}$ ONERA/DOTA, Université de Toulouse, F-31055 Toulouse France \\ *Email: jean-baptiste.dherbecourt@onera.fr
}

\begin{abstract}
We report on a direct detection differential absorption lidar (DIAL), designed for remote detection of $\mathrm{CH}_{4}$ and $\mathrm{CO}_{2}$. The system is based on a single-frequency optical parametric oscillator/amplifier system, tunable in the 1.57$1.65 \mu \mathrm{m}$ range. The DIAL system, called NAOMI GAZL, was tested on a controlled gas release facility in October 2018.
\end{abstract}

\section{INTRODUCTION}

Remote detection, localization, and quantification of gaseous species is a topic of high interest in the frame of various applications, from safety to environmental or industrial monitoring. In such context, differential absorption lidars (DIAL) are promising since they can be designed to address different species, especially using emitters in the infrared where most chemicals of interest display characteristic absorption bands.

In the frame of NAOMI project (for New Advanced Observation Method Integration), Onera and Total jointly investigated different remote sensing methods, to be able to probe gases such as $\mathrm{CO}_{2}$ and $\mathrm{CH}_{4}$ remotely. Several systems where developed, and tested on a specific gas leak simulation facility called TADI (for Transverse Anomaly Detection Infrastructure).

Here, we present a compact, range-resolved, multi-species DIAL for both $\mathrm{CO}_{2}$ and $\mathrm{CH}_{4}$, based a widely tunable, single frequency emitter, with a high conversion efficiency. Indeed, only few DIAL systems have been reported lately in the literature, for this kind of applications [1,2], and here we exploit a specific emitter architecture. The emitter is based on a pulsed, nanosecond, single frequency, nested cavity optical parametric oscillator (NesCOPO) [3], amplified to $16 \mathrm{~mJ}$, and implemented in a direct detection lidar system. This lidar is called NAOMI GAZL.

\section{METHODOLOGY}

\subsection{The NAOMI GAZL DIAL}

Basically, as it is well known, in a DIAL, at least two laser pulses are emitted, at two wavelengths called ON (in coincidence with one of the targeted species absorption line) and OFF (on an absorption minimum). The laser pulses are backscattered by the atmosphere, collected and detected by the DIAL receiver, and a gas plume can be localized and quantified along the line of sight by comparing the backscattered $\mathrm{ON}$ and OFF signals.

NAOMI GAZL emitter is based on an amplified NesCOPO. The core of the NesCOPO is a multitrack periodically-poled $\mathrm{LiNbO}_{3}$ (PPLN) crystal. Given the chosen poling periods, several lines in the 1.57-1.65 $\mu \mathrm{m}$ range can be addressed for $\mathrm{CO}_{2}$ and $\mathrm{CH}_{4}$ measurement (Fig.1). Water vapour lines are weak but could also be addressed.

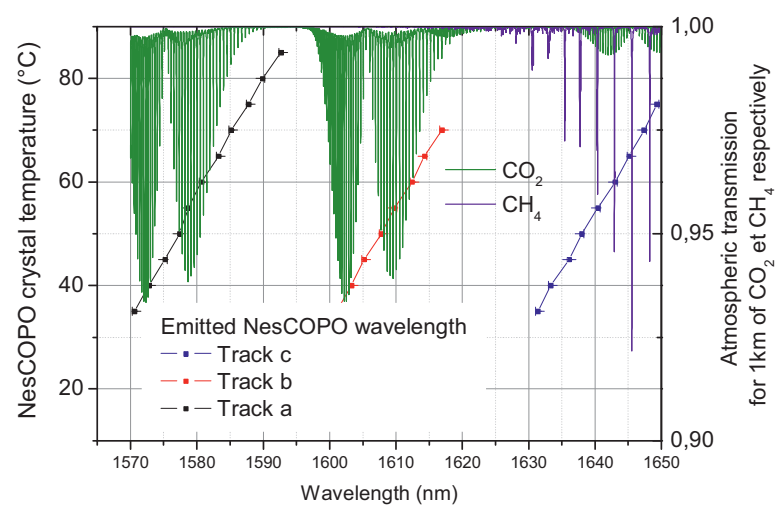

Fig. 1. NAOMI GAZL NesCOPO emitted wavelength as a function of the PPLN temperature, for three different poling periods a). The atmospheric transmission for $\mathrm{CO}_{2}$ (for $400 \mathrm{ppm}$ ) and $\mathrm{CH}_{4}$ (for 2 ppm) over $1 \mathrm{~km}$ are given. 
The NesCOPO is pumped by less than $1 \mathrm{~mJ}$ of a $10 \mathrm{~ns}, \quad 100 \mathrm{~Hz}$, injection-seeded Q-switched Nd:YAG laser (Innolas). An optical parametric amplification stage, based on a PPLN crystal followed by KTA amplifiers allows to emit up to $16 \mathrm{~mJ}$ of single frequency radiation in the 1.57 $1.65 \mu \mathrm{m}$ range, while pumped by around $100 \mathrm{~mJ}$.

NAOMI GAZL receiver unit is a custom Newtonian telescope, of $25 \mathrm{~cm}$ diameter, coupled to a commercial, $300 \mu \mathrm{m}$ diameter, amplified InGaAs PIN photodiode, coupled to an 8-bits digitizer. Given the chosen amplifier, the maximum detection bandwidth is $14.5 \mathrm{MHz}$, corresponding approximately to $10 \mathrm{~m}$ spatial range resolution cells along the line of sight.

\subsection{Preliminary testing on atmospheric $\mathrm{CH}_{4}$}

Prior to the system deployment on the TADI facility, the system was tested in ONERA facility in Palaiseau (France) in September 2018, on atmospheric $\mathrm{CH}_{4}$. For this measurement, the photodiode alignment was optimized for long range measurement, resulting in a $200 \mathrm{~m}$ blind zone. A typical measurement result is displayed in Fig.2. The obtained statistical errors (due to the detection noise only) are around $3 \mathrm{ppm}$, at a $1 \mathrm{~km}$ range, for integration times of $250 \mathrm{~s}$ and a range resolution of $150 \mathrm{~m}$, and less than $0.5 \mathrm{ppm}$ at a $300 \mathrm{~m}$ range. These performances are sufficient given the simulated gas leak tests foreseen on TADI platform. For more accurate environmental monitoring, improvement of these performances via further averaging or better detection using a commercially available avalanche photodiode can be expected.

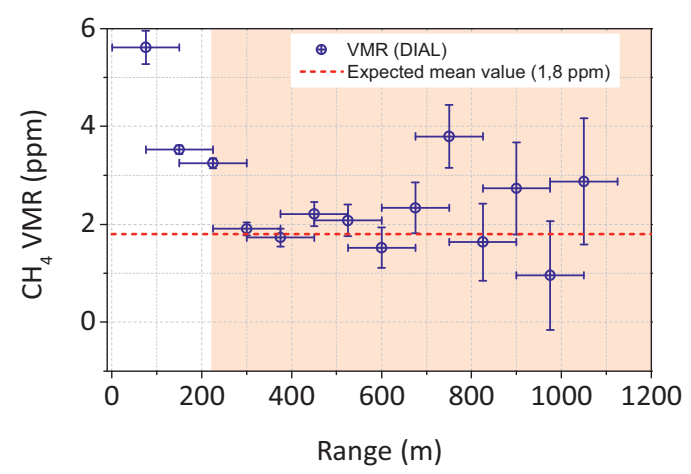

Fig. 2. NAOMI GAZL measurement for atmospheric $\mathrm{CH}_{4}$ : volume mixing ratio (VMR) as a function of the range, for an integration time of $250 \mathrm{~s}$ and a measurement resolution of $150 \mathrm{~m}$. The red dotted line corresponds to the expected $1.8 \mathrm{ppm}$ atmospheric concentration, and the white area corresponds to the lidar blind zone.

\section{OCTOBER 2018 TADI TEST CAMPAIGN}

\subsection{The TADI facility}

Located in Lacq (France), TADI facility is an open-air site measuring $2,000 \mathrm{~m}^{2}$ for qualifying systems designed to detect, localize and quantify remotely $\mathrm{CO}_{2}$ and $\mathrm{CH}_{4}$ releases. Equipped with surface facilities (pipelines, columns, recovered wellheads, etc.), this test area can reproduce around 30 scenarios of controlled emissions from 0.3 to $300 \mathrm{~g} / \mathrm{s}$ of gas in an industrial environment.

NAOMI GAZL was deployed on the TADI facility during a test campaign in October 2018 (see Fig. 3).

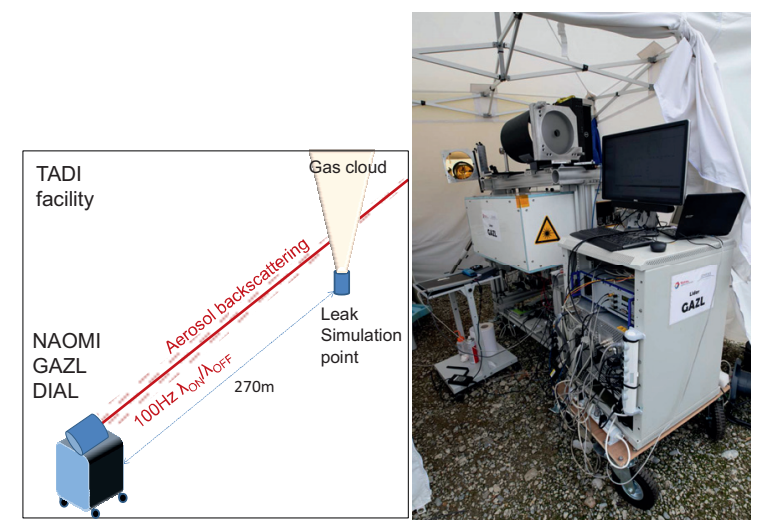

Fig. 3. NAOMI GAZL deployment scheme on TADI and picture of the system.

\subsection{Example of measurement results}

The NAOMI GAZL DIAL was located $250 \mathrm{~m}$ away from the gas release system. It was operated in a high resolution mode, i.e. with a spatial range resolution of $10 \mathrm{~m}$ (corresponding to the maximum detector amplifier bandwidth). Given the wind condition during these measurements, it was also operated on a high speed temporal mode with $10 \mathrm{~s}$ temporal averaging. Fig.4 a) and b) show an example of remote detection, quantification and localization of a $\mathrm{CH}_{4}$ plume, generated by TADI, using GAZL. The system was tested for different fluxes of $\mathrm{CH}_{4}$ and $\mathrm{CO}_{2}$. Comparisons with integrated concentration in ppm.m retrieved from LWIR imaging hyperspectral data acquired during this campaign can also be used then to analyse and validate GAZL results (Fig.4 c). 


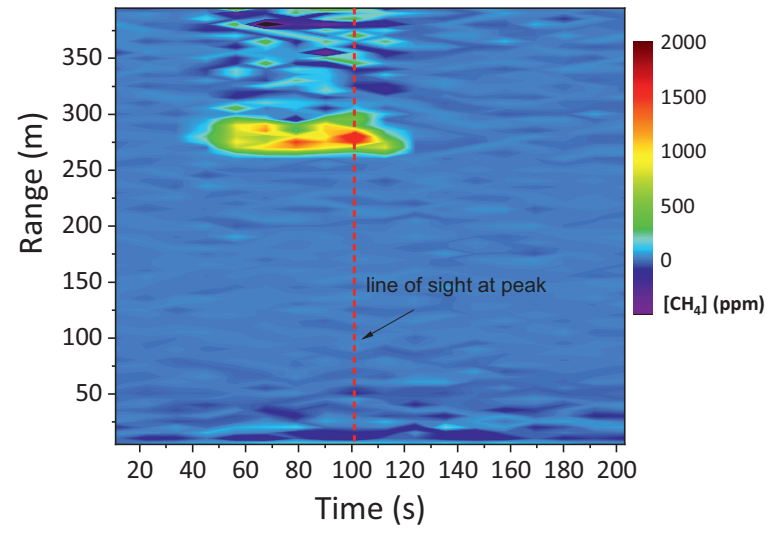

a)

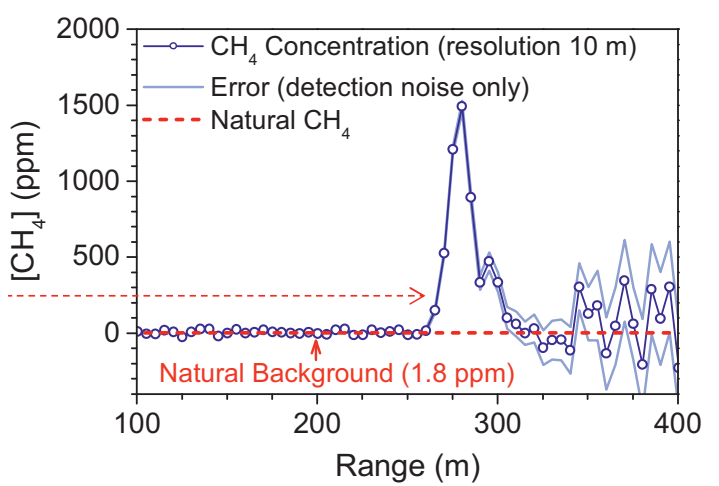

b)

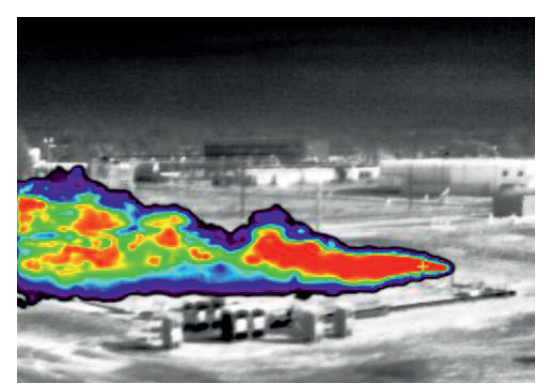

c)

Fig. 3. a) Example of measurement performed using GAZL DIAL, on TADI facility: a gas plume is generated $250 \mathrm{~m}$ away from the DIAL. The estimated $\mathrm{CH}_{4}$ plume concentration with a $10 \mathrm{~m}$ range resolution and a $10 \mathrm{~s}$ temporal resolution is shown on the left curve. In b), we can see a zoom of the $\mathrm{CH}_{4}$ concentration along the line of sight, with error bars corresponding to the detection noise. c) Methane plume quantitative image (range: $0-200.000 \mathrm{ppm} . \mathrm{m}$ ) from hyperspectral LWIR data at a distance of $100 \mathrm{~m}$ from the leak with a $5 \mathrm{~s}$ temporal resolution and a $15 \mathrm{~cm}$ spatial resolution.

\section{ACKNOWLEDGEMENTS}

This development was carried out in the frame of TOTAL and Onera NAOMI project.

\section{REFERENCES}

[1] G.A. Wagner, D.L. Plusquellic, Optics Express Vol.26, n 15,19420-19434 (2018)

[2] A. Amediek, G. Ehret, A. Fix, M. Wirth, C. Büdenbender, M. Quatrevalet, C. Kiemle, and C. Gerbig, Appl. Opt. 56, 5182-5197 (2017)

[3] A. Godard, M. Raybaut, M. Lefebvre, Encyclopedia of Analytical Chemistry (2017) 